2. Th. Skolem, Om konstitutionen av den identiske kalkuls grupper, Third Scand. Math. Congr., 1913, pp. 149-163.

3. —Über gewisse "Verbände" oder "Lattices," Avh. Norske Vid. Akad. Oslo (1936) pp. 1-16. 1940.

4. G. Birkhoff, Lattice theory, Amer. Math. Soc. Colloquium Publications, vol. 25,

5. R. Church, Numerical analysis of certain free distributive structures, Duke Math. J. vol. 6 (1940) pp. 7.32-734.

6. M. Ward, Bull. Amer. Math. Soc. Abstract 52-5-135.

Columbia University

\title{
ON COMPLETE LATTICES AND A PROBLEM OF BIRKHOFF AND FRINK ${ }^{1}$
}

\section{K. BALACHANDRAN ${ }^{2}$}

Two decomposition theorems for elements of complete lattices in terms of join prime or completely join prime elements are obtained (Theorems 1 and 2). The latter theorem gives a solution for a problem of Birkhoff and Frink on the relation between completely prime and completely join irreducible ideals. An interesting special case of this solution-for complemented lattices-is also noted (Theorem $3)$.

1. Definitions. An element $a$ of a lattice $L$ is called join irreducible if $a_{1} \cup a_{2}=a \rightarrow a_{1}$ or $a_{2}=a$, and join prime if $a_{1} \cup a_{2} \geqq a \rightarrow a_{1}$ or $a_{2} \geqq a$. Similarly $a$ is called completely join irreducible if (for all existing joins $\left.\bigcup_{i} a_{i}\right) \bigcup_{i} a_{i}=a \rightarrow$ some $a_{i}=a$, and completely join prime if $\cup_{i} a_{i} \geqq a$ $\rightarrow$ some $a_{i} \geqq a$.

The definitions of the corresponding dual concepts (indicated in each case by using the prefix "meet" in the place of "join") are quite clear.

A lattice $L$ will be said to be infinitely meet distributive if all (existing) meets $\bigcap_{i} a_{i}$ in it are distributive; the meet $\bigcap_{i} a_{i}$ is said to

Received by the editors February 26, 1953 and, in revised form, August 15, 1954.

1 The arrangement of the material in the present version follows the suggestions of the referee to whom the author's thanks are due. His thanks are also due to Dr. V.S. Krishnan with whom he has had useful consultations while preparing the manuscript.

2 Government of India Senior Research Scholar. 
be distributive if for arbitrary element $a$ there holds the equality: $a \cup\left(\bigcap_{i} a_{i}\right)=\bigcap_{i}\left(a \cup a_{i}\right)$.

The symbol $>$ will denote the usual covering relation so that $a>b$ means that $a>b$ and there is no element $x$ with $a>x>b$.

\section{Lemmas.}

Lemma 1. A join prime element of $L$ is join irreducible; conversely, if $L$ is distributive, a join irreducible element is join prime.

Proof. The first part follows from the definitions. For the second part, let $L$ be distributive and $a \in L$ be join irreducible. Then, if $a_{1} \cup a_{2} \geqq a, a=\left(a_{1} \cup a_{2}\right) \cap a=\left(a_{1} \cap a\right) \cup\left(a_{2} \cap a\right)$, so that, since $a$ is join irreducible, $a=a_{1} \cap a$ or $a_{2} \cap a$, i.e., $a \leqq a_{1}$ or $a_{2}$, whence $a$ is join prime.

Lemma 2. Every join prime element $p(\neq 0)$ of a complemented lattice is an atom. ${ }^{3}$

Proof. If $a<p$, and $a^{\prime}-$ a complement of $a$, then since $a \cup a^{\prime}=1 \geqq p$, $a \geqq p$, and $p$ is join prime, there results: $a^{\prime} \geqq p$, whence $a^{\prime} \geqq p>a$ so that $a=a \cap a^{\prime}=0$, or $p$ is an atom.

Lemma $3{ }^{4}$ Let $L$ be a complete, infinitely meet distributive lattice. Then if $a$ is such that $\left(\mathrm{C}_{1}\right) a>b \rightarrow a>b_{1} \geqq b$ for some $b_{1}, a$ is a join of completely join irreducibles.

Proof. It can be clearly supposed that $a$ itself is not completely join irreducible. Since 0 is completely join irreducible the join $b$ of all completely join irreducibles $\leqq a$ exists, and $b \leqq a$.

If possible, assume that $b<a$. Then by $\left(\mathrm{C}_{1}\right)$ there exists a $b_{1}$ with $b \leqq b_{1}<a$. If $x<a$ always implied $x \leqq b_{1}$, then $a$ itself would be completely join irreducible (for, $x_{i}<a \rightarrow x_{i} \leqq b_{1} \rightarrow \bigcup_{i} x_{i} \leqq b_{1}<a$ ). Hence there exists at least an $x$ with $x<a, x \leq b_{1}$.

Now let $c=\bigcap_{i} x_{i}$ be the meet of all $x<a, x \leq b_{1}$; then $c \$ b_{1}$. For, $c=\bigcap_{i} x_{i} \leqq b_{1}$ would imply: $b_{1}=b_{1} \cup\left(\bigcap_{i} x_{i}\right)=\bigcap_{i}\left(b_{1} \cup x_{i}\right)$; and since $b_{1} \prec a$, $b_{1} \leqq b_{1} \cup x_{i} \leqq a$, and $x_{i} \leqq b_{1}$, this gives $b_{1} \cup x_{i}=a$, so that $b_{1}=\cap a=a$-a contradiction (since $b_{1} \prec a$ ). Hence $c \$ b_{1}$, and so a fortiori $c \$ b$.

Next, if $y<c$, then $y<c<a$; again $y \leqq b_{1}$ (else $y$ would have to be an " $x_{i}$ " which is impossible, since $y<c \leqq x_{i}$ ). Hence $y \leqq c \cap b_{1}<c$ (since $\left.c \$ b_{1}\right)$. It follows that $c$ is completely join irreducible.

Thus, $c$ is a completely join irreducible element with $c \$ b, c \leqq a-$ which contradicts the definition of $b$, whence $b=a$, and the proof is complete.

\footnotetext{
${ }^{3}$ An element $a$ of a lattice with 0 is called an atom if $a \neq 0$, and $x<a \rightarrow x=0$.

- For a related result see [2, p. 304, Theorem 7] (citations in square brackets refer to the bibliography).
} 
LEMma 4. Let $L$ be a lattice in which every element is a join of a finite number of join primes. Then the lattice $L^{*}$ of ideals of $L$ is infinitely meet distributive; in particular, any existing meet $\bigcap_{i} a_{i}$ in $L$ is distributive.

Proof. For establishing the first assertion it is sufficient to prove the inequality: $\bigcap_{i}\left(A \cup B_{i}\right) \subseteq A \cup\left(\bigcap_{i} B_{i}\right)$ where $A, B_{i}$ are ideals of $L$.

If $x=\bigcup_{j=1}^{n} p_{j}$ (the $p_{j}$ 's being join prime elements) is an arbitrary element of $\bigcap_{i}\left(A \cup B_{i}\right)$, then of course $x \in\left(A \cup B_{i}\right)$ (for each $i$ ) so that $x$ has the form $x \leqq a_{i} \cup b_{i}\left(a_{i} \in A, b_{i} \in B_{i}\right)$. Since $p_{j} \leqq \bigcup_{j=1}^{n} p_{j}=x$ $\leqq a_{i} \cup b_{i}$, and $p_{j}$ is join prime, $p_{j} \leqq a_{i}$ or $b_{i}$, i.e., $p_{j} \in A$ or $B_{i}$. Hence $p_{j} \in A$, or $p_{j} \in$ every $B_{i}$ (and hence also $\in \bigcap_{i} B_{i}$ ). Thus (each) $p_{j} \in A$ $\cup\left(\cap_{i} B_{i}\right)$ so that $x=\bigcup_{j=1}^{n} p_{j} \in A \cup\left(\cap_{i} B_{i}\right)$, which proves the stated inequality, and thereby the first assertion.

The second assertion follows from the first by noting that the isomorphism between elements and principal ideals in $L$ preserves all existing meets of elements.

COROLlary. If $L$ is complete and every element of $L$ is a join of a finite number of join primes, then $L$ is infinitely meet distributive.

LemMa 5. If $L$ is a complete lattice in which every element is a meet of completely meet primes, then $L$ is infinitely meet distributive.

Proof. It suffices to prove the inequality $a \cup\left(\bigcap_{i} a_{i}\right) \geqq \bigcap_{i}\left(a \cup a_{i}\right)$, where $\bigcap_{i} a_{i}$ is any meet in $L$.

By hypothesis, $a \cup\left(\bigcap_{i} a_{i}\right)=\bigcap_{j} c_{j}$, where the $c_{j}$ 's are completely meet primes. Since (each) $c_{j} \geqq \bigcap_{j} c_{j}=a \cup\left(\bigcap_{i} a_{i}\right) \geqq \bigcap_{i} a_{i}$, and $c_{j}$ is completely meet prime, $c_{j} \geqq$ some $a_{i}$; also $c_{j} \geqq \bigcap_{j} c_{j} \geqq a$. Hence, $c_{j} \geqq a \cup a_{i}$ $\geqq \bigcap_{i}\left(a \cup a_{i}\right)$, and therefore $a \cup\left(\bigcap_{i} a_{i}\right)=\bigcap_{j} c_{j} \geqq \bigcap_{i}\left(a \cup a_{i}\right)$. Q.E.D.

Lemma 6. Every ideal of a lattice $L$ is a meet of completely meet irreducible ideals ${ }^{5}$ (cf. [2, p. 307, Theorem 11]).

Proof. Let $\bigcap_{i} A_{i}$ be the meet of all completely meet irreducible ideals $A_{i} \supseteq A$ (so that $\bigcap_{i} A_{i} \supseteq A$ ). If $\bigcap_{i} A_{i} \supset A$, there exists some element $a$ with $a \in \cap A_{i}, a \notin A$. By Zorn's lemma there is a maximal chain $C=\left(B_{j}\right)$ of ideals $B_{j}$ such that $B_{j} \supseteq A, B_{j} \boxplus a$. The join $B=\cup_{j} B_{j}$ (which is simply the set union of the $B_{j}$ 's on account of their linear ordering) also satisfies the same conditions, and hence belongs to $C$, since $C$ is maximal. Further, if an ideal $I \supset B, I$ lies outside the chain $C$ (since $B$ is the highest element of $C$ ); also $I \ni a$, as otherwise $I$ would have to belong to $C$ by virtue of maximality of $C$. Hence the meet

${ }^{5} \mathrm{By}$ a completely meet irreducible ideal of $L$ is meant a completely meet irreducible element of $L^{*}$ (the lattice of ideals). 
$\bigcap_{i} I_{i}$ of all ideals $I_{i} \supset B$ contains $a$, and so $\neq B$. That is, $B$ is completely meet irreducible.

Again since $B \supseteq A, B$ must be one of the $A_{i}$ 's so that $B \supset \bigcap_{i} A_{i} \ni a$; on the other hand since $B \in C, B \nsupseteq a$. This contradiction proves $\bigcap_{i} A_{i}=A$.

\section{The main theorems.}

THEOREM 1. Let $L$ have the zero element and satisfy the ascending chain condition. Then every element of $L$ is a join of a finite number of join primes if, and only if, $L$ is infinitely meet distributive.

Proof. Let $L$ be infinitely meet distributive. Since the ascending chain condition holds, it is clear that every element $a$ satisfies condition $\mathrm{C}_{1}$ (of Lemma 3). Hence by Lemma 3, $a=U_{i} c_{i}$, where $c_{i}$ are (completely) join irreducible, and therefore also join prime (Lemma 1). Again, in view of the chain condition there exists a finite subset $c_{i_{k}}(k=1, \cdots, n)$ of the $\left\{c_{i}\right\}$ such that $U_{k=1}^{n} c_{i_{k}}=U_{i} c_{i}$ (for otherwise there would be in $\left\{c_{i}\right\}$ some infinite chain $c_{1}\left\langle c_{1} \cup c_{2}<c_{1} \cup c_{2} \cup c_{3} \cdots\right.$, contradicting the chain condition). This completes the "if" part, while the "only if" part results from corollary to Lemma 4.

THEOREM 2. The following assertions concerning a complete lattice $L$ are all equivalent:

(i) Every element of $L$ is a join of a finite number of join primes.

(ii) $L$ is distributive and every element of $L$ is a join of a finite number of join irreducible elements.

(iii) The lattice $L^{*}$ of ideals of $L$ is infinitely meet distributive.

(iv) Every completely meet irreducible ideal of $L$ is a completely meet prime ideal. ${ }^{6}$

Proof. First, by Lemma 4, (i) implies that $L^{*}$ (and hence also $L$ ) is distributive; whereas, in a distributive lattice every join irreducible element is join prime (Lemma 1). Hence (i) and (ii) are equivalent.

Next, (i) implies (iii) by Lemma 4 . Again since in an infinitely meet distributive lattice every completely meet irreducible element is completely meet prime (cf. Lemma 1), (iii) implies (iv). Also (iv) implies (iii); for, Lemmas 6 and 4 give: Every ideal of $L$ is a meet of completely meet prime ideals, whence (by Lemma 5) $L^{*}$ is infinitely meet distributive.

The proof will be now completed by showing (iii) implies (i). Assume that (iii) holds, and let $A=P(a)$ be a principal ideal of $L$. Then $A \supset B \rightarrow A>B_{1} \supseteq B$, for some ideal $B_{1}$. For, in the set $S$ of ideals

\footnotetext{
- I.e., completely prime element of $L^{*}$.
} 
which $\supseteq B$ and $C A$, there exists by Zorn's Lemma (cf. proof of Lemma 6) a maximal $B_{1} \in S$, so that $A>B_{1} \supseteq B$.

Now Lemma 3 can be applied to $L^{*}$ (taking for $a$ any principal ideal $A=P(a))$. It then results that $A=P(a)=\bigcup_{i} A_{i}$, where $A_{i}$ are completely join irreducible ideals.

Since $A_{i}$ is the join of all principal ideals contained in it, $A_{i}$ itself must be a principal ideal (since $A_{i}$ is completely join irreducible); and moreover, if $A=P\left(a_{i}\right)$ the element $a_{i}$ is join irreducible, and so join prime (Lemma 1 ).

Thus, $A=P(a)=\cup_{i} P\left(a_{i}\right)$, whence $a \leqq a_{1} \cup \ldots \cup a_{n}$ and so $a=a_{1} \cup \ldots \cup a_{n}$ (since $a_{i} \leqq a$ )-which completes the proof.

4. Relation to a problem of Birkhoff and Frink. In their paper [2] Birkhoff and Frink have raised (on p. 313) the problem of finding all complete lattices $L$ in which $\left(\mathrm{C}_{2}\right)$ every completely meet irreducible dual ideal is completely prime. ${ }^{7}$ Theorem 2 (or more correctly its dual form formulated for dual ideals) furnishes some information on such lattices.

If in the above problem the complete lattices $L$ are further restricted to be complemented, then we obtain a surprisingly simple characterization for the lattices answering to the conditions of the problem: viz.,

THEOREM 3. The complete, complemented lattices $L$ satisfying the condition: every completely meet irreducible ideal is completely meet prime, are precisely the finite Boolean algebras.

Proof. That every finite Boolean algebra $B$ satisfies the stated condition $^{8}$ follows from Lemma 1 and the known fact that the lattice of ideals of a Boolean algebra is distributive.

Conversely, let $L$ be a complete, complemented lattice satisfying this condition. By Theorem 2 it results that (i) $L$ is distributive, (ii) each element $a$ is a join $a=\bigcup_{i=1}^{m} p_{i}$ of finite number of join prime elements $p_{i}$. Since $L$ is complemented, by Lemma $2, p_{i}$ is either 0 or is an atom. Thus, every element $(\neq 0)$ of $L$ is a join of a finite number of atoms.

${ }^{7}$ According to [2] a dual ideal $A$ is completely prime if $\bigcup_{i} a_{i} \in A \rightarrow$ some $a_{i} \in A$. Now let $A$ be completely prime dual ideal of a complete lattice $L$ and $A \supseteq \bigcap_{i} A_{i}$. Then $A \supseteq$ some $A_{i}$; else there would exist $a_{i} \in A_{i}, a_{i} \notin A$, so that $\bigcup_{i} a_{i} \in \bigcap_{i} A \subseteq A-$ contradicting the above definition. Hence for a complete lattice a completely prime dual ideal is the same as a completely meet prime dual ideal-in the sense of the present paper (cf. footnote 6).

${ }^{8}$ Note that since $B$ is finite the adjectives "completely" in the condition lose significance. 
Next, let $P=\left(p_{i}\right)$ denote the set of all join prime elements $\left(p_{i}=0\right.$, or is an atom). If $1=\bigcup_{i=1}^{m} p_{i}\left(p_{i} \neq 0\right)$ and $p(\neq 0)$ are in $P$, then $0<p \leqq p \cap\left(\cup_{i=1}^{m} p_{i}\right)=\bigcup_{i=1}^{m}\left(p \cap p_{i}\right)$, whence $p \cap p_{i} \neq 0$ (for some $\left.i=i_{1}\right)$. Hence, since $p, p_{i_{1}}$ are atoms, $p=p_{i_{1}}$. Thus, $P$ is the finite set $\left\{0, p_{1}, \cdots, p_{m}\right\}$, and since each element of $L$ is a join of elements from $P, L$ itself is finite. Finally, since $L$ is complemented (hypothesis) and distributive (proved), $L$ is a Boolean algebra. ( $L$ is, in fact, the finite Boolean algebra of all subsets of $P$.) The proof is complete.

Incidentally, the above theorem (in its dual form) corrects the erroneous assertion made by Birkhoff and Frink (loc. cit.) that every complete, atomic Boolean algebra $B$ satisfies the condition $\left(C_{2}\right)$. This assertion holds only in the case $B$ is finite.

\section{BIBLIOGRAPHY}

1. G. Birkhoff, Lattice theory, Amer. Math. Soc. Colloquium Publications, vol. 25, rev. ed., New York, 1948.

2. G. Birkhoff and O. Frink, Representations of lattices by sets, Trans. Amer. Math. Soc. vol. 64 (1948) pp. 299-316.

3. L. Nachbin, On a characterization of the lattice of all ideals of a Boolean ring, Fund. Math. vol. 36 (1949) pp. 137-142.

UNIVERSITY OF MADRAS 